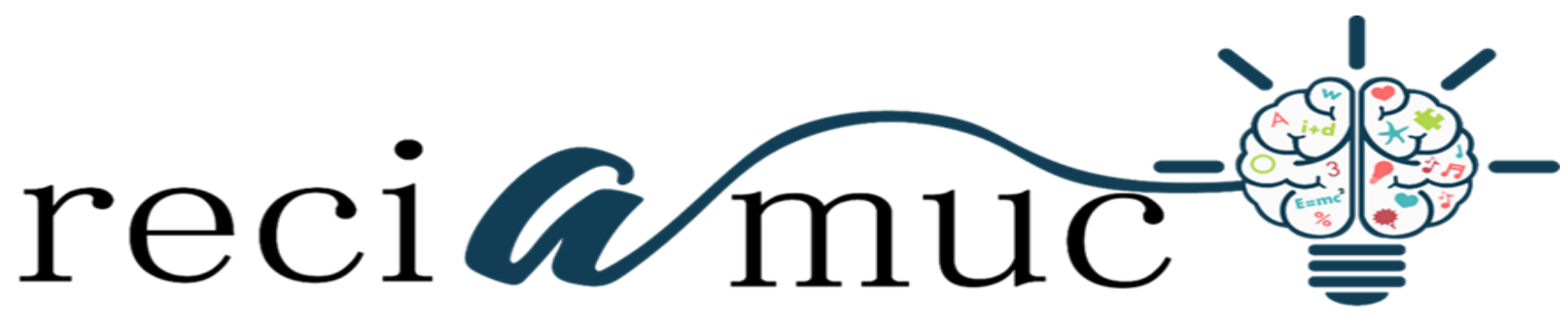

Revista cientifica de investigación actualización del mundo de las ciencias

\author{
Cristhian Michael Mendoza Aguilar a; Oswaldo Alexis Yagual Villon ${ }^{\text {b}}$; Cristhian \\ Jacinto Macas Quevedo ${ }^{c}$; María Judith Naranjo Escobar ${ }^{\text {d }}$
}

Tratamiento integral de pie diabético

Integral treatment of diabetic foot

Revista Científica de Investigación actualización del mundo de las Ciencias. Vol. 3 núm., 2, abril, ISSN: 2588-0748, 2018, pp. 170-195

DOI: $10.26820 /$ reciamuc/3.(2).abril.2019.170-195

URL: http://reciamuc.com/index.php/RECIAMUC/article/view/332

Código UNESCO: 3205 Medicina Interna

Tipo de Investigación: Artículo de Revisión

(c) RECIAMUC; Editorial Saberes del Conocimiento, 2019

Recibido: $15 / 01 / 2019$

Aceptado: 07/02/2019

Publicado: 01/04/2019

Correspondencia: emmamendoza1@outlook.com
a. Médico; Investigador Independiente; Guayaquil, Ecuador; emmamendoza1@outlook.com
b. Médico; Investigador Independiente; Guayaquil, Ecuador; oswaldoayv@ hotmail.com
c. Médico; Investigador Independiente; Guayaquil, Ecuador; drcristhianmacas@gmail.com
d. Médico; Investigador Independiente; Guayaquil, Ecuador; mariajudithnaranjo@gmail.com 


\section{Tratamiento integral de pie diabético}

Vol. 3, núm. 2., (2019)

Cristhian Michael Mendoza Aguilar; Oswaldo Alexis Yagual Villon; Cristhian Jacinto Macas

Quevedo; María Judith Naranjo Escobar

\section{RESUMEN}

La enfermedad del pie diabético es un problema de salud, que afecta a $15 \%$ de los 200 millones de pacientes con diabetes en el mundo. La amputación mayor, por encima o por debajo de la rodilla, es una complicación temida de la diabetes. Más del 60\% de las amputaciones no traumáticas en el mundo se llevan a cabo en la población diabética. Muchos pacientes que se someten a una amputación, tienen una historia de ulceración. Las amputaciones mayores aumentan la morbilidad y la mortalidad y reducen la calidad de vida del paciente. El tratamiento de las complicaciones del pie es uno de los principales elementos de la absorción de recursos económicos y de salud dirigida a la población diabética. Está claro que el tratamiento efectivo puede dar lugar a una reducción en el número de amputaciones mayores. En los últimos años, se ha visto un aumento significativo en el conocimiento acerca de las vías fisiopatológicas de esta complicación, junto con mejoras en las técnicas de diagnóstico, pero sobre todo un enfoque terapéutico conservador estandarizado, lo que permite la recuperación del miembro en un alto porcentaje de los casos. Este objetivo se ha logrado en centros especializados. Un avance importante para el tratamiento del pie diabético es el diagnóstico que difiere del pie neuropático y neuroisquémica. Esta diferenciación es esencial para un tratamiento eficaz. Una úlcera neuropática que no es tratada adecuadamente puede convertirse en una úlcera crónica que no se cura. La neuroartropatía de Charcot es una complicación particular de neuropatía que puede conducir a la fragmentación o la destrucción de las articulaciones y los huesos. En la población diabética la enfermedad vascular periférica (PVD) es el principal factor de riesgo de amputación. Si la enfermedad vascular periférica es ignorada, el tratamiento quirúrgico de la lesión no puede tener éxito.

Palabras clave: Diabetes, Pie Diabético, Amputación, Ulceración Diabética, Isquemia Crítica, Cirugía Pie Diabético. 


\title{
Tratamiento integral de pie diabético
}

Vol. 3, núm. 2., (2019)

Cristhian Michael Mendoza Aguilar; Oswaldo Alexis Yagual Villon; Cristhian Jacinto Macas Quevedo; María Judith Naranjo Escobar

\begin{abstract}
Diabetic foot disease is a health problem, which affects $15 \%$ of the 200 million patients with diabetes in the world. Major amputation, above or below the knee, is a dreaded complication of diabetes. More than $60 \%$ of nontraumatic amputations in the world are carried out in the diabetic population. Many patients who undergo an amputation have a history of ulceration. Major amputations increase morbidity and mortality and reduce the patient's quality of life. The treatment of foot complications is one of the main elements of the absorption of economic and health resources aimed at the diabetic population. It is clear that effective treatment can lead to a reduction in the number of major amputations. In recent years, there has been a significant increase in knowledge about the pathophysiological pathways of this complication, together with improvements in diagnostic techniques, but above all a standardized conservative therapeutic approach, which allows the recovery of the limb in a High percentage of cases. This objective has been achieved in specialized centers. An important advance for the treatment of diabetic foot is the diagnosis that differs from the neuropathic and neuroischemic foot. This differentiation is essential for effective treatment. A neuropathic ulcer that is not treated properly can become a chronic ulcer that does not heal. Charcot's neuroarthropathy is a particular complication of neuropathy that can lead to fragmentation or destruction of joints and bones. In the diabetic population, peripheral vascular disease (PVD) is the main risk factor for amputation. If peripheral vascular disease is ignored, the surgical treatment of the lesion cannot be successful.
\end{abstract}

Key words: Diabetes, Diabetic Foot, Amputation, Diabetic Ulceration, Critical Ischemia, Diabetic Foot Surgery. 


\section{Tratamiento integral de pie diabético}

Vol. 3, núm. 2., (2019)

Cristhian Michael Mendoza Aguilar; Oswaldo Alexis Yagual Villon; Cristhian Jacinto Macas

Quevedo; María Judith Naranjo Escobar

\section{Introducción.}

El pie diabético debe ser considerado como un síndrome que afecta un porcentaje significativo de los pacientes con diabetes, el autor (Edmonds, 2010) afirma que se reconocen dos aspectos importantes en esta enfermedad: el pie neuropático y el pie neuroisquémica. Ambos tipos tienen diferentes momentos fisiopatológicos, fases y resultados de diagnóstico terapéutica.

Estos dos tipos de ulceras son distintas e implican dos marcos de tiempo, lo que justifica una atención metodológicamente integrada, pero esencialmente diferentes. En 1990, Pecoraro esbozó las vías que llevan a un sujeto diabético con complicaciones neuropáticos e isquémicos través de la cadena de eventos definidos para desarrollar una progresión infectiva que por consiguiente conduce a un riesgo elevado de tener que someterse a la amputación (Pecoraro, Reiber, \& Burgess, 1990). Es útil para aclarar la importancia de definir los pasos esenciales en las estrategias de diagnósticos y terapéuticas en el tratamiento del pie diabético destinadas a salvar la extremidad. Es solamente mediante el reconocimiento de los factores capaces de influir negativamente en el pronóstico y corregirlos (por ejemplo, isquemia crítica y de revascularización, osteomielitis y su tratamiento quirúrgico, síndrome compartimental, cirugía de emergencia) que pueden reducir el número de amputaciones en la población diabética.

Tomando en consideración que la ulceración en pie neuropático es debido al estrés biomecánico y alta presión, que implica la superficie plantar de los dedos del pie y las cabezas de los metatarsianos. El tratamiento de una úlcera plantar neuropático debe corregir las presiones plantares patológicos, a través, de alivio de la carga de peso. El tratamiento quirúrgico de las deformidades, con o sin ulceraciones, son una terapia eficaz. Mientras que la neuroartropatía 


\section{Tratamiento integral de pie diabético}

Vol. 3, núm. 2., (2019)

Cristhian Michael Mendoza Aguilar; Oswaldo Alexis Yagual Villon; Cristhian Jacinto Macas Quevedo; María Judith Naranjo Escobar

Charcot es una complicación particular de neuropatía que puede conducir a la fragmentación o la destrucción de las articulaciones y los huesos.

El Grupo de Trabajo Internacional del pie diabético ha desarrollado recientemente un sistema de clasificación para fines de investigación llamado Sistema PEDIS, en donde las úlceras se clasifican en cinco categorías: la perfusión, alcance / tamaño, profundidad / pérdida de tejido, infección y la sensibilidad

En 1989, la Declaración de San Vicente citó, entre sus objetivos más importantes, la reducción en un 50\% del número de amputaciones mayores en 5 años consecutivos. Esto se sumó a la mejora de la calidad de la atención en todo el mundo para los pacientes afectados por la diabetes Mellitus, aunque algunas cifras de población de amputación no han mostrado una caída en los pacientes diabéticos, los informes de Suecia, Dinamarca, Italia y el Reino Unido han demostrado una reducción de amputación mayor. (Edmonds \& Forster, 2001)

Durante los últimos 20 años, han aumentado progresivamente conocimientos y métodos de tratamiento fisiopatológicos para el pie diabético. El porcentaje de aumento va enfocado en la salvación de la extremidad en pacientes tratados en unidades multidisciplinares está vinculada a técnicas probadas de tratamiento de un pie con infección aguda, pie neuropático, y las condiciones de isquemia crítica de pie neuroisquémica. (Reiber, Lipsky, \& Gibbons, 2012).

Algún conocimiento fisiopatológico notable en relación con el desarrollo de las úlceras ha sido importante en la puesta en práctica de ciertos comportamientos terapéuticos que, a su vez, han 


\section{Tratamiento integral de pie diabético}

Vol. 3, núm. 2., (2019)

Cristhian Michael Mendoza Aguilar; Oswaldo Alexis Yagual Villon; Cristhian Jacinto Macas

Quevedo; María Judith Naranjo Escobar

demostrado ser particularmente eficaces reduciendo la posibilidad de amputación y brindando una mayor calidad de vida a los pacientes

Por tanto, el objetivo de esta revisión es definir estrategias terapéuticas en los distintos tipos de síndrome del pie diabético. Se han propuesto varios sistemas de clasificación para clasificar las úlceras del pie diabético. Los sistemas de clasificación más populares han sido durante mucho tiempo el Wagner y Texas Universidad Clasificaciones. (Armstrong, Lavery, \& Harkless, Validación de un sistema de clasificación de la herida diabética: contribución de la profundidad, la infección y la enfermedad vascular con el riesgo de amputación., 2008)

\section{Métodos y materiales.}

Para el desarrollo de este proceso investigativo, se plantea como metodología la encaminada hacia una orientación científica particular que se encuentra determinada por la necesidad de indagar en forma precisa y coherente una situación, en tal sentido (Davila, 2015) define la metodología "como aquellos pasos previos que son seleccionados por el investigador para lograr resultados favorables que le ayuden a plantear nuevas ideas”. (p.66)

Lo citado por el autor, lleva a entender que el desarrollo de la acción investigativa busca simplemente coordinar acciones enmarcadas en una revisión bibliográfica con el fin de complementar ideas previas relacionadas Tratamiento integral de pie diabético, a través de una revisión de literatura, para así finalmente elaborar un cuerpo de consideraciones generales que ayuden a ampliar el interés propuesto. 


\section{Tratamiento integral de pie diabético}

Vol. 3, núm. 2., (2019)

Cristhian Michael Mendoza Aguilar; Oswaldo Alexis Yagual Villon; Cristhian Jacinto Macas Quevedo; María Judith Naranjo Escobar

\section{Tipo de Investigación.}

Dentro de toda práctica investigativa, se precisan acciones de carácter metodológico mediante las cuales, se logra conocer y proyectar los eventos posibles que la determinan, así como las características que hacen del acto científico un proceso interactivo ajustado a una realidad posible de ser interpretada. En este sentido, se puede decir, que la presente investigación corresponde al tipo documental, definido por Castro (2016), "se ocupa del estudio de problemas planteados a nivel teórico, la información requerida para abordarlos se encuentra básicamente en materiales impresos, audiovisuales y /o electrónicos”. (p.41).

En consideración a esta definición, la orientación metodológica permitió la oportunidad de cumplir con una serie de actividades inherentes a la revisión y lectura de diversos documentos donde se encontraron ideas explicitas relacionadas con los tópicos encargados de identificar a cada característica insertada en el estudio. Por lo tanto, se realizaron continuas interpretaciones con el claro propósito de revisar aquellas apreciaciones o investigaciones propuestas por diferentes investigadores relacionadas con el tema de interés, para luego dar la respectiva argumentación a los planteamientos, en función a las necesidades encontradas en la indagación.

\section{Fuentes Documentales.}

El análisis correspondiente a las características que predomina en el tema seleccionado, llevan a incluir diferentes fuentes documentales encargadas de darle el respectivo apoyo y en ese sentido cumplir con la valoración de los hechos a fin de generar nuevos criterios que sirven de referencia a otros procesos investigativos. Para (CASTRO, 2016) las fuentes documentales 


\section{Tratamiento integral de pie diabético}

Vol. 3, núm. 2., (2019)

Cristhian Michael Mendoza Aguilar; Oswaldo Alexis Yagual Villon; Cristhian Jacinto Macas

Quevedo; María Judith Naranjo Escobar

incorporadas en la investigación documental o bibliográfica, "representa la suma de materiales sistemáticos que son revisados en forma rigurosa y profunda para llegar a un análisis del fenómeno" (p.41). Por lo tanto, se procedió a cumplir con la realización de una lectura previa determinada para encontrar aquellos aspectos estrechamente vinculados con el tema, con el fin de explicar mediante un desarrollo las respectivas apreciaciones generales de importancia.

\section{Técnicas para la Recolección de la Información.}

La conducción de la investigación para ser realizada en función a las particularidades que determinan a los estudios documentales, tiene como fin el desarrollo de un conjunto de acciones encargadas de llevar a la selección de técnicas estrechamente vinculadas con las características del estudio. En tal sentido, (Bolívar, 2015), refiere, que es "una técnica particular para aportar ayuda a los procedimientos de selección de las ideas primarias y secundarias”. (p. 71).

Por ello, se procedió a la utilización del subrayado, resúmenes, fichaje, como parte básica para la revisión y selección de los documentos que presentan el contenido teórico. Es decir, que mediante la aplicación de estas técnicas se pudo llegar a recoger informaciones en cuanto a la revisión bibliográfica de los diversos elementos encargados de orientar el proceso de investigación. Tal como lo expresa, (Bolívar, 2015) "las técnicas documentales proporcionan las herramientas esenciales y determinantes para responder a los objetivos formulados y llegar a resultados efectivos" (p. 58). Es decir, para responder con eficiencia a las necesidades investigativas, se introdujeron como técnica de recolección el método inductivo, que hizo posible llevar a cabo una valoración de los hechos de forma particular para llegar a la explicación desde una visión general. 


\section{Tratamiento integral de pie diabético}

Vol. 3, núm. 2., (2019)

Cristhian Michael Mendoza Aguilar; Oswaldo Alexis Yagual Villon; Cristhian Jacinto Macas Quevedo; María Judith Naranjo Escobar

Asimismo, se emplearon las técnicas de análisis de información para la realización de la investigación que fue ejecutada bajo la dinámica de aplicar diversos elementos encargados de determinar el camino a recorrer por el estudio, según, (Bolívar, 2015) las técnicas de procesamiento de datos en los estudios documentales "son las encargadas de ofrecer al investigador la visión o pasos que debe cumplir durante su ejercicio, cada una de ellas debe estar en correspondencia con el nivel a emplear" (p. 123). Esto indica, que para llevar a cabo el procesamiento de los datos obtenidos una vez aplicado las técnicas seleccionadas, tales como: fichas de resumen, textual, registros descriptivos entre otros, los mismos se deben ajustar al nivel que ha sido seleccionado.

\section{Resultados.}

La enfermedad del pie diabético es un problema de salud, que afecta a $15 \%$ de los 200 millones de pacientes con diabetes en el mundo. Es recurrente que existan amputaciones en los miembros inferiores a causa de los cuidados no debidos de esta enfermedad. Más del 60\% de las amputaciones no traumáticas en el mundo se llevan a cabo en la población diabética, la incidencia de amputaciones mayores varía de 0,5 a 5 por cada 1000 pacientes. Muchos pacientes que se someten a una amputación, tienen una historia de ulceración que pudo haberse tratado a tiempo, ya que, las amputaciones mayores aumentan la morbilidad y la mortalidad y reducen la calidad de vida del paciente.

Alrededor de $15 \%$ de los diabéticos han tenido una úlcera de pie en algún momento de sus vidas. La incidencia y prevalencia de la úlcera diabética en la literatura varía, dependiendo de la población y el método de encuesta utilizada. Estudios llevados a cabo en el Reino Unido han puesto 


\section{Tratamiento integral de pie diabético}

Vol. 3, núm. 2., (2019)

Cristhian Michael Mendoza Aguilar; Oswaldo Alexis Yagual Villon; Cristhian Jacinto Macas

Quevedo; María Judith Naranjo Escobar

de manifiesto una prevalencia de esta lesión entre 5,3\% y 7,4\% (Walters, Gatling, \& Mullee, 2012).

En los EE.UU., Ramsey puso de relieve una incidencia acumulada de 5,8\% de las lesiones ulceradas en pacientes dados de alta del hospital durante un período de 3 años. En Suecia se registró una incidencia anual de 3,6\% de ulceración, y en Holanda se demostró una incidencia anual de 2,1\% de las úlceras en pacientes diabéticos de tipo II. En un amplio estudio de la comunidad en el Reino Unido, la incidencia anual de la ulceración del pie era un poco más de 2,0\% entre todos los pacientes y entre 5,0\% y 7,5\% entre los pacientes con neuropatía periférica. (Abbot, Vileykite, Williamson, Carrington, \& Boulton, 2016)

Las tasas de amputación varían según los países, grupos raciales, y dentro de los países, y pueden superar el 20 por 100.000 personas. Las ulceraciones y sobre todo amputaciones se agravan por el pronóstico incorrecto, las tasas de morbilidad y mortalidad son más altas en la población con ulceraciones. La mortalidad en el período peri-operatorio es alta: $9 \%$ según un estudio holandés y el 10-15\% en el Reino Unido. La tasa de mortalidad dentro de los 30 días de una amputación mayor (por encima o debajo de la rodilla), ha alcanzado el 10\%. En un estudio de seguimiento de una población amputada, se ha demostrado una tasa de supervivencia a 5 años del 50\%. (Faglia, Favales, \& Morabito, 2001)

\section{Fisiopatología y tratamiento de Pie Neuropático}

La Neuropatía se asocia con un 8 a 18 veces más riesgo de ulceración y una de 2 a 15 veces mayor riesgo de amputación. La neuropatía periférica es considerada la alteración fisiopatológica básica que lleva a neuroartropatía de Charcot. Los mecanismos mediante los cuales la neuropatía actúa como un acontecimiento patogénico de ulceración y por lo tanto a la amputación son 


\section{Tratamiento integral de pie diabético}

Vol. 3, núm. 2., (2019)

Cristhian Michael Mendoza Aguilar; Oswaldo Alexis Yagual Villon; Cristhian Jacinto Macas Quevedo; María Judith Naranjo Escobar

complejos y diferentes. Por encima de todo, la reducción de la sensibilidad de protección (incluyendo la sensibilidad al dolor y calor) conduce a una reducción en la percepción de los estímulos del dolor. Por otra parte, el componente de motor de la neuropatía implica un debilitamiento progresivo del componente muscular intrínseco compuestos de interósea y los músculos lumbricales. Se revela como una deformación en flexión del dedo del pie y la formación de zonas plantares sobrecargados, identificables debajo de las cabezas de los metatarsianos y las puntas de los dedos de los pies. En tercer lugar, ha sido ampliamente demostrado que una alteración de pie biomecánico, que incluye aumento de presión plantares, anomalías óseas, limitaciones de movilidad, y equinus, están vinculados a un aumento significativo en el riesgo de ulceración.

Un paciente desarrolla una lesión neuropático debido a las presiones plantares patológicas. La sobrecarga patológica se detecta inmediatamente en un pie que mantiene la sensibilidad completa. Sin embargo, en un sujeto neuropático, que presenta traumas ambientales (zapatos que no le calzan bien, exposición a objetos afilados y a superficies ambientales) pasan desapercibidos. Por consiguiente, la úlcera se desarrolla debido a la falta de percepción del trauma repetido vinculado al movimiento. Las características clínicas de la lesión neuropática son los siguientes: desarrollo de un espacio sobrecargado rodeado de una formación insensible antes del desarrollo de la lesión, sin síntomas dolorosos. Al igual que las úlceras por presión, las lesiones plantares neuropáticas tienden a ser socavado, con una pequeña abertura hacia la superficie de la piel, en comparación con la verdadera participación de los tejidos más profundos.

El riesgo de la lesión es el empeoramiento en términos de destrucción del tejido profundo progresivo y la infección, está vinculada a la coexistencia de un componente isquémico. Por lo 


\section{Tratamiento integral de pie diabético}

Vol. 3, núm. 2., (2019)

Cristhian Michael Mendoza Aguilar; Oswaldo Alexis Yagual Villon; Cristhian Jacinto Macas

Quevedo; María Judith Naranjo Escobar

tanto, la enfermedad vascular periperal debe ser excluida en la evaluación inicial de una lesión ulcerada con características clínicas adecuadas a las de una lesión neuropático. El desbridamiento adecuado debe seguir la evaluación de una úlcera, se debe eliminar por completo el callo que rodea la lesión y todos los tejidos no saludables, hasta que los bordes sangrantes saludables son revelados. El desbridamiento quirúrgico permite la eliminación completa de todo el material necrótico y disminuye la carga bacteriana, promoviendo así la curación. Entonces es necesario llevar a cabo una "sonda al hueso" precisa maniobra con el fin de establecer la participación de las estructuras más profundas, como los tendones, cápsulas articulares y los huesos. (Karchmer \& Gibbons, 2014)

Sin embargo, los autores (Remedios, Valabhji, Oelbaum, Sharp, \& Mitchell) expresan que en la mayoría de los casos, la maniobra de sonda-hueso con un objeto contundente estéril es suficiente para diagnosticar osteomielitis. Por tanto, es solamente necesario usar métodos más complejos (tales como resonancia magnética nuclear y / o de barrido de leucocitos radiomarcado) en un pequeño porcentaje de los casos.

La literatura destaca claramente cómo la descarga es esencial en los casos de lesión neuropático plantar. Las técnicas de descarga simples son múltiples e incluyen moldes y botas, sandalias, zapatos de la mitad de los apósitos de espuma o fieltro. El uso de un molde no desprendible ha demostrado recientemente por (Caravaggi, 2007) ser un tratamiento más rápido para las úlceras neuropáticas plantares que un zapato de medio (Fig. 1) 


\section{Tratamiento integral de pie diabético}

Vol. 3, núm. 2., (2019)

Cristhian Michael Mendoza Aguilar; Oswaldo Alexis Yagual Villon; Cristhian Jacinto Macas Quevedo; María Judith Naranjo Escobar

\section{Figura $N^{a}$ 1. Preparación de un molde no extraíble.}
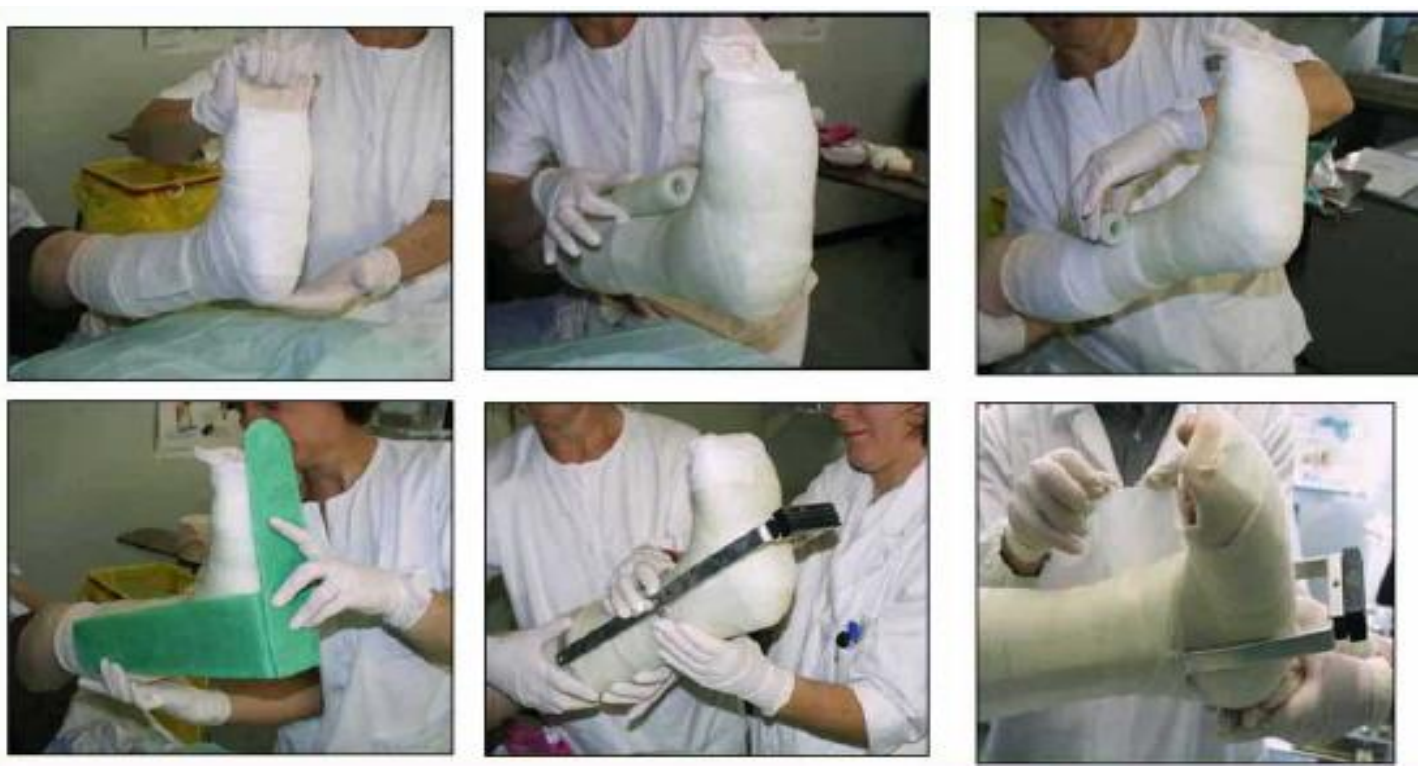

Fuente: (Caravaggi, 2007).

El mejor efecto está probablemente relacionado a la mayor adherencia terapéutica de un dispositivo no extraíble, lo que provoca la reducción de la carga de peso y los tiempos de caminar en comparación con otros dispositivos.

No obstante, (Piagges, Viacava, Rizzo, \& L, 2003) han demostrado recientemente que el componente inflamatorio de lesiones ulceradas, quirúrgicamente eliminado después del tratamiento con un yeso en la pierna, se redujo en comparación con otros tipos de tratamiento. Además, los parámetros de la reparación de tejidos (producción de colágeno, la angiogénesis, la cantidad de tejido de granulación) fueron mejores en el grupo tratado con los moldes de contacto total. 


\section{Tratamiento integral de pie diabético}

Vol. 3, núm. 2., (2019)

Cristhian Michael Mendoza Aguilar; Oswaldo Alexis Yagual Villon; Cristhian Jacinto Macas

Quevedo; María Judith Naranjo Escobar

Las contraindicaciones absolutas al uso de un TCC (Yeso de contacto completo) incluyen isquemia con transcutánea de oxígeno a presión (TcPO2) de menos de 30-50 mmHg medidos en el aspecto dorsal de la parte delantera del pie, infección activa, la participación de los tejidos y / o osteomielitis profundo, y dificultades para caminar debido a neurológica complicaciones y / o hypovision. Tratamos a pacientes con bajo peso molecular heparinas de forma concomitante.

Recientemente se ha informado de la eficiencia de otros dispositivos de Descarga tales como Aircast ${ }^{\circledR}$ DESMONTABLE WALKER (Armstrong \& Laver, 2005) ya que, la planificación de diagnóstico incorrecto, montaje incorrecto de la herida, y por lo tanto un enfoque terapéutico erróneo, implican un mayor riesgo de que la herida se vuelva crónica y un elevado riesgo de propagar la infección.

El primer paso en el tratamiento de una lesión neuropática ulcerada sin complicaciones es el desbridamiento local, vestirse, y fuera de la carga. Sin embargo, existen situaciones clínicas en las que la cirugía se convierte en el tratamiento de elección. Los autores (Armstrong \& Frykberg, 2003) han proporcionado una clasificación de la cirugía del pie diabético que correlaciona clases de tratamiento con una puntuación de riesgo de amputación. Las indicaciones para el tratamiento quirúrgico de las úlceras neuropáticas plantares son esencialmente: 1) coexistencia de osteomielitis 2) exostosis plantar que pone herida cicatrizada en un alto riesgo de recurrencia 3) heridas crónicamente ulceradas resistente a la terapia conservadora. 


\section{Tratamiento integral de pie diabético}

Vol. 3, núm. 2., (2019)

Cristhian Michael Mendoza Aguilar; Oswaldo Alexis Yagual Villon; Cristhian Jacinto Macas Quevedo; María Judith Naranjo Escobar

\section{Neuroartropatía de Charcot}

La neuroartropatía de Charcot es sin duda la más clara denominación del papel dominante que la neuropatía puede jugar en la pérdida fisiológica progresiva en el pie de un paciente diabético. Eichenholtz codificó las fases de desarrollo (ver tabla 1) según (Armstrong \& Frykberg, 2003) aparecen varias zonas afectadas en una clasificación de 5 etapas (Fig. 2).

\section{Tabla Nº 1. Fases Eichenholtz en el pie de Charcot.}

\begin{tabular}{|c|l|}
\hline Etapa 0 & $\begin{array}{l}\text { "En riesgo" de pie y tobillo. Paciente con diabetes y la neuropatía periférica que ha tenido un } \\
\text { esguince o una fractura aguda. }\end{array}$ \\
\hline Nivel 1 & $\begin{array}{l}\text { Desarrollo. Los pacientes se presentan con un proceso inflamatorio agudo; Los rayos X muestran } \\
\text { la fragmentación del hueso con los desechos y la interrupción conjunta ocasional o dislocación. }\end{array}$ \\
\hline Etapa 2 & $\begin{array}{l}\text { La coalescencia. Hinchazón, calor y regresión enrojecimiento, y los rayos X muestran el hueso } \\
\text { que rodea la articulación como esclerótica; absorción de residuos finos se produce, y la mayoría } \\
\text { de los grandes fragmentos se fusionan juntos. }\end{array}$ \\
\hline Etapa 3 & $\begin{array}{l}\text { Reconstrucción. Continúa resolución de la inflamación, y los rayos X muestran remodelación } \\
\text { persistente con un poco de reforma de la arquitectura conjunta. Este es el mejor momento para la } \\
\text { fusión quirúrgica. }\end{array}$ \\
\hline
\end{tabular}

Fuente: (Armstrong \& Frykberg, 2003). 


\section{Tratamiento integral de pie diabético}

Vol. 3, núm. 2., (2019)

Cristhian Michael Mendoza Aguilar; Oswaldo Alexis Yagual Villon; Cristhian Jacinto Macas

Quevedo; María Judith Naranjo Escobar

\section{Figura N². Clasificación de Armstrong y Frykberg del pie de Charcot.}

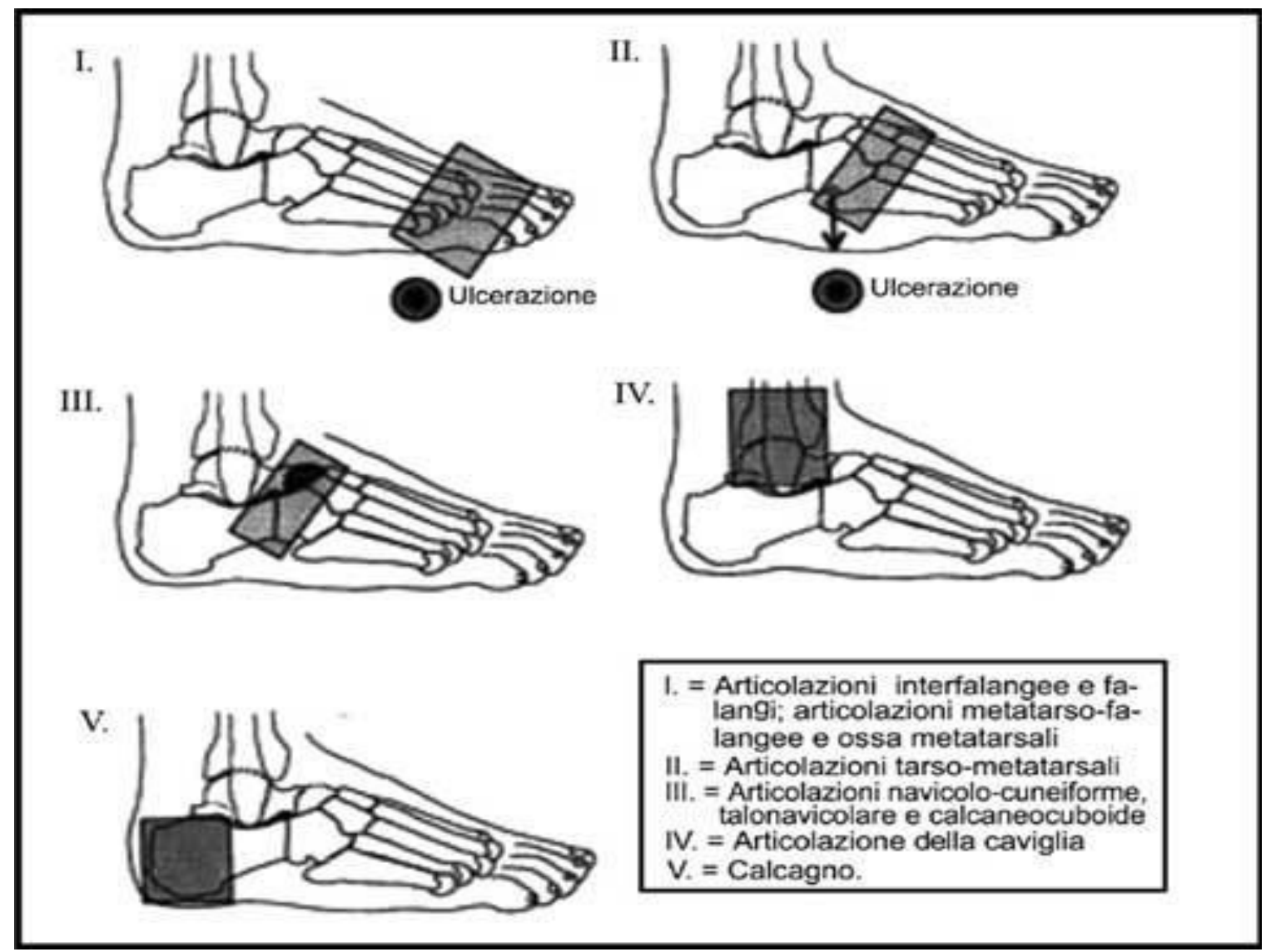

Fuente: (Armstrong \& Frykberg, 2003).

Los objetivos del tratamiento son: 1) para crear y mantener una planta del pie estable; 2) para sanar una herida ulcerada; 3) para curar fracturas; 4) para evitar deformaciones.

La elección del tratamiento depende de la etapa Eickenholtz y localización de la enfermedad. El tratamiento de elección del pie de Charcot agudo es la inmovilización prolongada. Los principios de este enfoque son el control y el tratamiento de edema, proporcionando estabilidad esquelética y la protección de los tejidos blandos. El tiempo de inmovilización varía dependiendo del sitio. Para la parte media del pie, se pronostica 10-12 semanas de la descarga total, seguido por otros 4-10 semanas de soporte de peso protegido con un yeso o una bota. Se 


\section{Tratamiento integral de pie diabético}

Vol. 3, núm. 2., (2019)

Cristhian Michael Mendoza Aguilar; Oswaldo Alexis Yagual Villon; Cristhian Jacinto Macas Quevedo; María Judith Naranjo Escobar

refiere a la parte posterior del pie, 10-12 semanas de la descarga total seguida de 6-12 meses de soporte de peso protegido. Una vez que se ha alcanzado la estabilización clínica, el paciente debe ser tratado con el calzado preventivo especial.

En el caso de heridas superficiales (grado I-II de la clasificación de Texas), el tratamiento de elección se basa en descargar presiones patológicas, junto con apósitos locales, que varían dependiendo del estado de la herida. Una vez que la herida ha sanado, se toma la decisión de si colocar al paciente en un programa de prevención o no. A través de la educación, las plantillas y las plantas inferiores del eje de balancín, el paciente puede mantener el riesgo de infección recurrente bajo control. Si el paciente tiene que ser operado, esto tiende a minimizar el riesgo de ulceraciones recurrentes.

Cuando la herida ulcerada implica articulaciones y / o los huesos, está indicado el tratamiento quirúrgico, por lo general que implica la eliminación de la lesión, la exposición y la limpieza de exostosis. Los huesos implicados son generalmente los de la cara medial del pie, el navicular y / o cuneiforme y / o la base del primer metatarsiano medial; en el aspecto lateral del pie, huesos implicados son la base del quinto metatarsiano o cuboides.

Principios de tratamiento del pie Neuro-Isquémico.

Las características epidemiológicas de la enfermedad vascular periférica (PVD) son más evidentes en los diabéticos que en la población general [57-59]. La principal característica de la enfermedad vascular periférica en los diabéticos es la presentación clínica y morfológica. (Reiber E. , 2016) 


\section{Tratamiento integral de pie diabético}

Vol. 3, núm. 2., (2019)

Cristhian Michael Mendoza Aguilar; Oswaldo Alexis Yagual Villon; Cristhian Jacinto Macas

Quevedo; María Judith Naranjo Escobar

Las obstrucciones se encuentran principalmente debajo de la rodilla; las oclusiones prevalecen en comparación con la estenosis, los síntomas dolorosos se reducen a menudo o ausente, debido a la coexistencia de sensibilidad neuropático, y calcinosis arterial medial (MAC) común. Estas características hacen de PVD en los diabéticos más difícil de diagnosticar y la terapia más problemática que en los no diabéticos. También significa que los PVD juega un papel fundamental en el pronóstico de amputación mayor. Sin embargo, desde la década de 1990, los procedimientos revascularizacion han demostrado ser opciones viables en comparación con el pensamiento inicial. Procedimientos que van desde la revascularización distal a la angioplastia y de derivación, todas las interrelaciones e intervenciones han sido capaces de cambiar el pronóstico original de amputación. (Aldeghi, 2005)

El problema fundamental de PVD diabética es la precisión del diagnóstico. Estos pacientes a menudo sufren poco o ningún dolor al caminar o en reposo. La presencia frecuente de calcificaciones arteriales es otro elemento confuso, a veces dando lugar a una evaluación incorrecta de la importancia de los parámetros de presión, ya que, tanto el tobillo a la presión y el índice tobillo-brazo. Estas características típicas de los diabéticos son los factores principales que conducen a la subestimación de la presencia de PVD. Este error juega un papel importante en la curación de heridas retardada y posible gangrena, y es un factor que contribuye a muchas amputaciones. (Lepantalo M, 2010). Esto es cierto en el caso de amputaciones menores cuando el pie carece de suficiente flujo de sangre, las heridas no se pueden curar, y una amputación es necesario a un nivel más proximal. 


\section{Tratamiento integral de pie diabético}

Vol. 3, núm. 2., (2019)

Cristhian Michael Mendoza Aguilar; Oswaldo Alexis Yagual Villon; Cristhian Jacinto Macas Quevedo; María Judith Naranjo Escobar

Ciertamente la revascularización endoluminal o quirúrgica es el único tratamiento capaz de reducir el número de amputaciones mayores significativamente, La revascularización puede restaurar el flujo arterial directo donde se ha interrumpido o reducido de manera significativa. Esta es una condición indispensable para la curación de una herida en un pie isquémico sin recurrir a la amputación. Este procedimiento es esencial en los casos de dolor en reposo. Es de vital importancia cuando se necesita la cirugía correctiva de una herida de la parte del pie. Consideramos que es incorrecto para realizar la amputación quirúrgica sin necesidad de llevar a cabo un diagnóstico exhaustivo de PVD y (en su caso) sin tener en cuenta revascularización. (La Fontaine J, 2001)

Pie infectado: Tiempo y protocolo terapéutico en el infarto agudo e infección crónica.

Una herida ulcerada puede comenzar como un caso no complicado, pero la infección puede desarrollar y llevar a un compromiso de los tejidos blandos, e incluso la afectación ósea. En sujetos diabéticos esto es debido al desarrollo de áreas plantares con hiperpresión, principalmente en la parte delantera del pie, mientras que en los casos neuroisquémicas es debido al contacto con el medio ambiente o al calzado inadecuado. Los casos de grave destrucción de los tejidos blandos, osteomielitis, síndrome compartimental e (infección progresiva a través de compartimentos plantares y dorsales) son verdaderas emergencias médicas y quirúrgicas.

A continuación, se presenta una tabla en donde se observan las características clínicas del pie diabético cuando presenta infección. 
Cristhian Michael Mendoza Aguilar; Oswaldo Alexis Yagual Villon; Cristhian Jacinto Macas

Quevedo; María Judith Naranjo Escobar

\section{Tabla N². Características clínicas de pie diabético Infecciones}

\begin{tabular}{|l|c|}
\hline \multicolumn{1}{|c|}{ Las manifestaciones clínicas de las infecciones } & Gravedad \\
\hline Herida sin purulencia o cualquier signo de inflamación & No-Infectada \\
\hline $\begin{array}{l}\text { Manifestación >2cm de la inflamación (purulencia o eritema, dolor, sensibilidad, } \\
\text { calor, induración) pero cualquier celulitis / eritema se extiende <2 cm alrededor de la } \\
\text { úlcera y la infección se limita a tejidos superficiales. No complicaciones locales o } \\
\text { enfermedad sistémica. }\end{array}$ & Templado \\
\hline $\begin{array}{l}\text { Infección en un paciente que está sistémicamente bien y metabólicamente estable } \\
\text { pero que tiene 1 de los siguientes síntomas: celulitis extendida }>2 \mathrm{~cm} \text {; linfangitis } \\
\text { extendido debajo de la fascia; absceso de tejido profundo; gangrena; músculo,tendón, } \\
\text { articulación, hueso involucrado. }\end{array}$ & Moderado \\
\hline $\begin{array}{l}\text { Infección en pacientes con toxicidad sistémica o inestabilidad metabólica (p. Ej., } \\
\text { Fiebre, escalofríos, taquicardia, hipotensión, confusión, vómitos, leucocitosis, } \\
\text { acidosis, hiperglucemia) }\end{array}$ & Grave \\
\hline
\end{tabular}

Fuente: (Eneroth M, 2014).

En estos casos, la fase isquémica no es un elemento inicial de riesgo de ulceración, pero sin duda es el caso más desfavorable para determinar el pronóstico de riesgo de amputación.

\section{Tratamiento de la infección}

La infección de los tejidos blandos, el compromiso progresivo de los tejidos profundos, y el desarrollo de focos osteomieliticos son los puntos que separan el tratamiento conservador de un abordaje quirúrgico más agresivo. Esta etapa debe incluir la planificación terapéutica cuidado, que debe basarse en un examen microbiológico después de la exclusión de la isquémica com-ponente. Claramente revascularización debe posponerse hasta después de tratamiento agudo de la infección.

Las infecciones que no suponen una amenaza inmediata de pérdida de la extremidad se definen como 'extremidad no en peligro', y se caracterizan generalmente por la ausencia de signos de sistémica intoxicación. En una celulitis de lesión superficial de $>2 \mathrm{~cm}$ generalmente no está presente, ni son abscesos profundos, osteomielitis o gangrena. Las infecciones definidas como 


\section{Tratamiento integral de pie diabético}

Vol. 3, núm. 2., (2019)

Cristhian Michael Mendoza Aguilar; Oswaldo Alexis Yagual Villon; Cristhian Jacinto Macas Quevedo; María Judith Naranjo Escobar

'extremidades mortales' muestran celulitis extendidas, abscesos profundos, osteomielitis o gangrena. La isquemia caracteriza a una lesión superficial como la extremidad amenazante proporcionando una clasificación más específica de la infección, como se muestra en la tab.3 (Lipsky, 2001). Las úlceras infectadas generan importantes signos y síntomas locales y generales en los diabéticos.

\section{Tabla N³. Recomendaciones de la evaluación de un paciente diabético con una infección del pie.}

\begin{tabular}{|l|}
\hline Describir la lesión (celulitis, úlceras, etc.) y cualquier drenaje (seroso, purulento, etc) \\
\hline Enumerar presencia o ausencia de diversos signos de inflamación \\
\hline Averiguar si o no la infección está presente, y el intento de definir causa probable \\
\hline Examinar los tejidos blandos para la evidencia de crepitus, abscesos, fístulas \\
\hline $\begin{array}{l}\text { Sonda de cualquier rotura de la piel con sonda de metal estéril para ver si el hueso se } \\
\text { puede llegar }\end{array}$ \\
\hline $\begin{array}{l}\text { Mida el (x anchura longitud; estimar la profundidad) de la herida; considerar } \\
\text { fotografía }\end{array}$ \\
\hline Palpar pulsos y registro de pedal; utilizar Doppler instrumento si es necesario
\end{tabular}

Fuente: (Lipsky, 2001).

En la mayoría de los estudios clínicos el tratamiento con antibióticos no mejora el resultado de las úlceras no infectadas. Un seguimiento, incluyendo el seguimiento cercano de las condiciones locales, es necesario, para asegurar que los signos y / o síntomas de infección local peligrosos están resaltados. El diagnóstico de la infección es clínico. La presencia de secreciones purulentas de dos o más signos de inflamación (eritema, calor, textura, dureza) se deben utilizar en el diagnóstico de una infección. Ante un caso clínico de infección no amenazante de la extremidad, lo mejor es 


\section{Tratamiento integral de pie diabético}

Vol. 3, núm. 2., (2019)

Cristhian Michael Mendoza Aguilar; Oswaldo Alexis Yagual Villon; Cristhian Jacinto Macas

Quevedo; María Judith Naranjo Escobar

comenzar el tratamiento con antibióticos desde el principio. Para infecciones leves, el tratamiento con antibiótico se administra por vía oral. El tratamiento oral es menos costoso, más fácil de manejar y por lo general suficiente para este tipo de pacientes. El tratamiento parenteral (dificultad en la absorción intestinal, alergias gastrointestinales, el aislamiento de las bacterias resistentes a la terapia antibiótica oral) solamente se puede elegir en algunos casos. El antibiótico elegido debe alcanzar buenos niveles de serosas y proporcionar una buena cobertura frente a las bacterias.

\section{Conclusiones.}

Durante el desarrollo del trabajo investigativo se observaron, los componentes de evaluación y el tratamiento que pueden ayudar a asegurar una curación exitosa y rápida de las úlceras del pie en pacientes diabéticos. Estos enfoques deben usarse siempre que sea posible para reducir la alta morbilidad y el riesgo de complicaciones graves que resulten de las úlceras del pie como lo son las amputaciones. Todavía hay mucho margen de mejora tanto en los tipos de técnicas utilizadas como en la garantía de que los médicos brinden el estándar de atención actual más alto. Sin embargo, se han creado clínicas especializadas multidisciplinarias, que combinan la experiencia de muchos tipos de proveedores de atención médica, incrementando mejoras en los resultados para las personas diabéticas con úlceras del pie.

De esta manera los caminos de diagnóstico y tratamientos examinados durante la investigación son sin duda, el fruto del enfoque multidisciplinario. La mejor manera de aumentar la prevención y el tratamiento de pacientes con complicaciones del pie diabético es a través de un equipo multidisciplinario independiente que se dedica exclusivamente a pacientes con esta patología. 


\section{Tratamiento integral de pie diabético}

Vol. 3, núm. 2., (2019)

Cristhian Michael Mendoza Aguilar; Oswaldo Alexis Yagual Villon; Cristhian Jacinto Macas Quevedo; María Judith Naranjo Escobar

Hoy en día existen las llamadas clínicas del pie que se enfrenta a situaciones de diferentes características dependiendo del entorno sanitario en el que los diversos especialistas trabajan. Específicamente son profesionales dedicados al problema de la diabetes y los expertos en las técnicas de prevención y tratamiento. Tomando en consideración, que el papel de la coordinación es asignado a un diabetólogo, con la colaboración de cirujanos vasculares y ortopédicos, radiólogos, cardiólogos, podólogos y enfermeras especializadas. La organización de la atención debe ofrecer la posibilidad de tratar las heridas no complicadas en un ambiente de sala, donde se utilizan técnicas modernas de descarga, terapia local y técnicas avanzadas.

La prevención de la recurrencia de la úlcera sigue siendo un desafío clínico importante, como lo demuestran las tasas de recurrencia que van del $28 \%$ a los 12 meses y del $100 \%$ a los 40 meses. Después de la curación completa, los pacientes deben cambiar lentamente la actividad completa y el soporte de peso, utilizando la terapia adecuada y calzado con plantillas personalizadas. Los zapatos de balancín rígidos reducen efectivamente la presión plantar del antepié. Algunos estudios de zapatos terapéuticos han demostrado que reducen la recurrencia de la úlcera Las pautas para la prescripción de calzado no están bien estandarizadas, y pocos practicantes miden la presión plantar en sitios de úlceras previas para asegurar que el calzado reduzca las altas presiones. La evidencia disponible sugiere que, además del calzado apropiado, la provisión de educación paciente y el cuidado regular del pie (incluido el desbridamiento de callosidades) pueden ayudar a prevenir las úlceras recurrentes.

Finalmente se puede concluir, que al realizar el tratamiento adecuado a las ulceras de pie diabético se evitará el riesgo de amputación ofreciendo mayor calidad de vida a los pacientes con 


\section{Tratamiento integral de pie diabético}

Vol. 3, núm. 2., (2019)

Cristhian Michael Mendoza Aguilar; Oswaldo Alexis Yagual Villon; Cristhian Jacinto Macas

Quevedo; María Judith Naranjo Escobar

esta patología teniendo en cuenta que la falta de curación reduce la calidad de vida tanto del paciente como del cuidador.

\section{Bibliografía.}

Abbot, Vileykite, Williamson, Carrington, \& Boulton. (2016). Multicenter study of the incidence of and predictive risk factors for diabetic neuropathic foot ulceration. Washington: Diab Care .

Aldeghi, F. F. (2005). Cambio en la mayor tasa de amputación en un centro dedicado al cuidado del pie diabético durante la década de 1980: los determinantes pronósticos para una amputación mayor. Complicaciones de la Diabetes, (págs. 80-100). Bogotá.

Armstrong, \& Frykberg. (2003). La clasificación de la cirugía del pie diabético: hacia una definición racional. Diabet Med.

Armstrong, \& Lavery. (2005). Evaluación de las ortesis de marcha e inamovible en la cicatrización de las heridas del pie diabético: un ensayo controlado aleatorio. Washington: Cuidado Diab.

Armstrong, Lavery, \& Harkless. (2008). Validación de un sistema de clasificación de la herida diabética: contribución de la profundidad, la infección y la enfermedad vascular con el riesgo de amputación. Buenos Aires: Diabetes Care.

Bolívar, J. (2015). Investigación Documental. México. Pax.

Caravaggi, C. (2007). Eficacia y seguridad de una fibra de vidrio no extraíbles fuera de cojinete fundido frente shoein terapéutica el tratamiento de úlceras de pie neuropático. Bogota: Diab Care.

Castro, J. (2016). Técnicas Documentales. México. Limusa. 


\section{Tratamiento integral de pie diabético}

Vol. 3, núm. 2., (2019)

Cristhian Michael Mendoza Aguilar; Oswaldo Alexis Yagual Villon; Cristhian Jacinto Macas Quevedo; María Judith Naranjo Escobar

Davila, A. (2015). Diccionario de Términos Científicos. Caracas: Editorial Oasis.

Edmonds, \& Forster. (2001). La reducción de las amputaciones en el diabético una estrategia para "tomar el control” con tratamiento conservador, así como la revascularización. Vasa.

Edmonds, M. (2010). Los avances en el cuidado del pie diabético. Mexico: The Lancet.

Eneroth M, L. J. (2014). Deep foot infections in patients with diabetes and foot ulcer: an entity with different characteristics, treatments, and prognosis. Diab Comp.

Faglia, Favales, \& Morabito. (2001). New ulceration, new major amputation and survival rates in diabetics subjects hospitalised for foot ulceration. Washington: Diab Care.

Karchmer, \& Gibbons. (2014). Infecciones en los pies en la diabetes: evaluación y gestión. 1-22.

La Fontaine J, R. A. (2001). El papel de la revascularización en amputaciones transmetatarsiana. Lima: J Am Med Assoc Podiatr.

Lepantalo M, B. F. (2010). Tukiainen E. Nunca amputan sin consulta de un cirujano vascular. Diab Metab Res Rev 2000; 7-64.

Lipsky. (2001). Infectious problems of the foot in diabetic patients in: Bowker JH, Pfeifer MA (eds): Levin and O'Neal's The Diabetic Foot 6th edition Mosby.

Pecoraro, R., Reiber, G., \& Burgess, E. (1990). [2]. Caminos a la amputación de la extremidad diabética. Bases para la prevención. Medellin: Diabetes Care.

Piagges, Viacava, Rizzo, \& L. (2003). Análisis semicuantitativo de las características histopatológicas de la úlcera en el pie neuropático: efectos de alivio de presión. Guatemala: Diab Care.

Reiber, E. (2016). La epidemiología de los problemas del pie diabético. Buenos Aires: Diabet Med. 
Vol. 3, núm. 2., (2019)

Cristhian Michael Mendoza Aguilar; Oswaldo Alexis Yagual Villon; Cristhian Jacinto Macas

Quevedo; María Judith Naranjo Escobar

Reiber, G., Lipsky, B., \& Gibbons, G. (2012). La carga de las úlceras del pie diabético. Montreal: Am J Surg.

Remedios, Valabhji, Oelbaum, Sharp, \& Mitchell. Tecnanocoloide para la evaluación de la osteomielitis en pies neuropático diabético. Mexico: Clin Radiol.

Walters, Gatling, \& Mullee. (2012). The distribution and severity of diabetic foot disease: a community study with comparison to non-diabetic group. Diabet Med.

$$
\text { (9) }(1)(0)
$$

RECONOCIMIENTO-NOCOMERCIAL-COMPARTIRIGUAL

CC BY-NC-SA

ESTA LICENCIA PERMITE A OTROS ENTREMEZCLAR, AJUSTAR Y CONSTRUIR A PARTIR DE SU OBRA CON FINES NO COMERCIALES, SIEMPRE Y CUANDO LE RECONOZCAN LA AUTORÍA Y SUS NUEVAS CREACIONES ESTÉN BAJO UNA LICENCIA CON LOS MISMOS TÉRMINOS. 